\title{
Serum levels of soluble CD163 and CXCL5 may be predictive markers for immune-related adverse events in patients with advanced melanoma treated with nivolumab: a pilot study
}

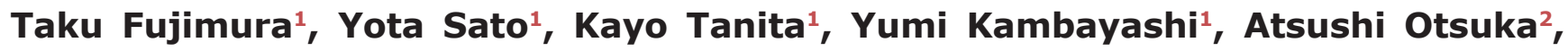
Yasuhiro Fujisawa ${ }^{3}$, Koji Yoshino ${ }^{4}$, Shigeto Matsushita ${ }^{5}$, Takeru Funakoshi ${ }^{6}$, Hiroo Hata7, Yuki Yamamoto ${ }^{8}$, Hiroshi Uchi ${ }^{9}$, Yumi Nonomura ${ }^{2}$, Ryota Tanaka ${ }^{3}$, Megumi Aoki $^{5}$, Keisuke Imafuku${ }^{7}$, Hisako Okuhira ${ }^{8}$, Sadanori Furudate ${ }^{1}$, Takanori Hidaka ${ }^{1}$ and Setsuya Aiba ${ }^{1}$

\footnotetext{
${ }^{1}$ Department of Dermatology, Tohoku University Graduate School of Medicine, Sendai, Japan

${ }^{2}$ Department of Dermatology, Kyoto University Graduate School of Medicine, Kyoto, Japan

${ }^{3}$ Department of Dermatology, Faculty of University of Tsukuba, Tsukuba, Japan

${ }^{4}$ Department of Dermatology, Tokyo Metropolitan Cancer and Infectious Disease Center Komagome Hospital, Tokyo, Japan

${ }^{5}$ Department of Dermato-Oncology/Dermatology, National Hospital Organization Kagoshima Medical Center, Kagoshima, Japan

${ }^{6}$ Department of Dermatology, Keio University School of Medicine, Tokyo, Japan

${ }^{7}$ Department of Dermatology, Hokkaido University Graduate School of Medicine, Sapporo, Japan

${ }^{8}$ Department of Dermatology, Wakayama Medical University, Wakayama, Japan

${ }^{9}$ Department of Dermatology, Kyushu University Graduate School of Medicine, Fukuoka, Japan

Correspondence to: Taku Fujimura, email: tfujimural@mac.com

Keywords: sCD 163; CXCL5; TAMs; melanoma; POSTN

Received: September 01, 2017 Accepted: February 10, 2018 Epub: February 15, 2018 Published: March 20, 2018

Copyright: Fujimura et al. This is an open-access article distributed under the terms of the Creative Commons Attribution License 3.0 (CC BY 3.0), which permits unrestricted use, distribution, and reproduction in any medium, provided the original author and source are credited.
}

\section{ABSTRACT}

Antibodies against PD-1, such as nivolumab and pembrolizumab, are widely used in the treatment of various cancers including advanced melanoma. The antiPD-1 Ab significantly prolongs survival in patients with metastatic melanoma, and its administration in combination with local or systemic therapy may also lead to improved outcomes. Although anti-PD-1 Ab-based combined therapy might be effective for the treatment of advanced melanoma, the associated risk of irAEs is an important consideration. Therefore, being able to predict irAEs is of great interest to oncologists. The purpose of this study was to evaluate the value of using serum levels of SCD163 and CXCL5 to predict irAEs in patients with advanced melanoma who were administered nivolumab. To this end, we analyzed these serum levels in 46 cases of advanced melanoma treated with nivolumab. In addition, the tumor stroma was evaluated by immunohistochemistry and immunofluorescence. We measured the serum levels of SCD163 and CXCL5 on day 0 (immediately before nivolumab administration) and day 42. The serum absolute levels of SCD163 were significantly increased in patients who developed AEs $(p=0.0018)$. Although there was no significant difference in serum levels of CXCL5, the absolute value of CXCL5 could at least be a supportive marker for the increased absolute levels of serum SCD163. This study suggests that SCD163 and CXCL5 may serve as possible prognostic biomarkers for irAEs in patients with advanced melanoma treated with nivolumab. 


\section{INTRODUCTION}

The program cell death-1/programmed death-ligand 1 (PD-1/PD-L1) pathway plays a critical role in the tumor immune response; thus, anti-PD-1 antibodies (Abs), such as nivolumab and pembrolizumab, are widely used in the treatment of various cancers including advanced melanoma [1-3]. The anti-PD-1 Ab significantly prolongs survival in patients with metastatic melanoma, and its administration in combination with local [4-8] or systemic therapy (e.g., ipilimumab, epacadostat) [2, 9] may also lead to improved outcomes. Although anti-PD-1 Ab-based combined therapy might prove effective for the treatment of advanced melanoma, the associated risk of immunerelated adverse events (irAEs) such as severe hepatitis, interstitial pneumonia, colitis, type 1 diabetes mellitus, hypophysitis, or myasthenia gravis is an important consideration [2]. Therefore, the prediction of irAEs is of great interest among oncologists.

Tumor-associated macrophages (TAMs) are characterized by their heterogeneity and plasticity, and may be functionally reprogrammed to polarized phenotypes by exposure to cancer-related factors, stromal factors, or infection, leading to the production of various chemokines that are specific to each cancer [10-14]. Concerning metastatic melanoma, periostin (POSTN) was found to be expressed in the region surrounding melanoma cell nests in the metastatic melanoma lesions of wounded mice and humans [15], suggesting that it might stimulate TAMs to produce chemokines that induce melanoma-specific tumor-infiltrating lymphocytes (TILs) in melanoma patients with systemic inflammation. As we previously reported, upon POSTN stimulation, M2 macrophages produce several immunosuppressive and autoimmune-related chemokines including CXCL5 [14]. Notably, CXCL5 is a biomarker of T helper 17 cellmediated autoimmune diseases such as multiple sclerosis, rheumatoid arthritis, and pemphigus vulgaris [16-18]; and soluble CD163 (sCD163) is a TAM marker that appears in the serum as a result of proteolytic shedding [19]. We previously reported that both CXCL5 and SCD163 might predict irAEs in 17 patients with advanced melanoma patients treated with nivolumab $[8,20]$. In this report, we further analyzed the serum levels of sCD163 and CXCL5 in 46 cases of advanced melanoma treated with nivolumab.

\section{RESULTS}

\section{Patients}

We collected data from 46 patients treated with nivolumab (Table 1). The mean patient age was 66.5 years (range, 31-93 years) and 52.8\% were males. The most common site of the primary tumor was in the extremities (39.1\%), followed by mucosal origin (30.4\%), trunk
(15.2\%), head/neck (6.5\%), and the ocular region (2.2\%). A dose of $2 \mathrm{mg} / \mathrm{kg}$ nivolumab was administered to the patients followed by 3 weeks of rest. The incidence of AE development was $45.7 \%$ (Grade 4: 6.5\%, Grade 3: 15.2\%, Grade 2: $19.6 \%$, Grade 1: $6.5 \%$ ), and the average onset of irAE development was 136 days (range, 21-370).

\section{Serum levels of sCD163 and CXCL5}

To determine whether serum levels of sCD163 and CXCL5 may predict AEs in patients treated with nivolumab, we evaluated their levels in 46 patients with advanced melanoma treated with nivolumab. Compared to baseline (day 0), the absolute value of sCD163 serum levels at day 42 were significantly increased in the group that developed irAEs compared with the group lacking irAEs ( $p=0.0018$; Figure 1A), whereas there was no significant difference in the absolute value of serum CXCL5 (Figure 1B). The increase or decrease of serum sCD163 and CXCL5 in each patient (Table 1) and each irAE (Table 2) is described. The cut-off point was determined using Youden's index. The threshold of the change rate of serum $\mathrm{SCD} 163$ was $\pm 21.3 \%$, while the threshold of the change rate of serum CXCL5 was $\pm 35.6 \%$. The sensitivity of serum $\mathrm{sCD} 163$ was $72.7 \%$, whereas that of CXCL5 was $50 \%$. The specificity of serum sCD163 was $75.0 \%$, whereas that of CXCL5 was 81.8\% (Figure 1C).

\section{Tissue samples, immunohistochemistry, and immunofluorescence}

Because sCD163 is an activation marker for $\mathrm{CD} 163^{+}$tissue macrophages [27], CXCL5 is produced from $\mathrm{CD} 163+$ macrophages by POSTN stimulation [14], and POSTN in melanoma is augmented by nonspecific inflammation such as wound healing [15], we hypothesized that the administration of nivolumab in advanced melanoma might increase the expression of POSTN in melanoma tissue, leading stimulated CD163 macrophages to produce sCD163 and CXCL5. To prove our hypothesis, we performed immunohistochemical staining for POSTN and CD163 in five patients with intransit advanced melanoma without irAEs, and in two patients with in-transit advanced melanoma with irAEs. The expression of POSTN in in-transit melanoma was augmented in stromal fibroblasts in patients with irAE, especially after nivolumab administration (Figure 2A). In contrast, the expression of POSTN in in-transit melanoma from irAE-developed patients before administration of nivolumab (Figure 2B) and in-transit melanoma from non-irAE-developed patients (Figure 2C) was low in fibroblasts distributed in peritumoral lesions. The semiquantitative analysis of POSTN expression is described in Table 3. CD163+ cells were detected in each group (Figure 2D). The number of CD163+ cells significantly increased 
Table 1: Patient characteristics and serum levels of sCD163 and CXCL5

\begin{tabular}{|c|c|c|c|c|c|c|c|c|}
\hline & Age & Sex & Onset of irAE & Location & sCD163 (ng/ml) & CXCL5 (pg/ml) & $\operatorname{ir} \mathbf{A E}$ & Grade \\
\hline 1 & 68 & $\mathrm{M}$ & 237 & Extremities & 115.782943 & 35.56162835 & hypophisitis & 4 \\
\hline 2 & 36 & M & 55 & Extremities & -44.23121476 & -17.57753725 & hepatitis & 4 \\
\hline 3 & 61 & $\mathrm{M}$ & 245 & Extremities & -11.4747151 & 174.0938997 & interstitial pneumonia & 4 \\
\hline 4 & 83 & $\mathrm{~F}$ & 370 & Extremities & 40.72768869 & -31.97872991 & radiation dermatitis & 3 \\
\hline 5 & 85 & $\mathrm{~F}$ & 27 & Other/Unknown & -25.44524995 & -91.17233749 & $\begin{array}{c}\text { chronic inflammatory } \\
\text { demyelinating } \\
\text { polyneuropathy }\end{array}$ & 3 \\
\hline 6 & 66 & $\mathrm{~F}$ & 214 & Ocular & 43.18056154 & -22.79478559 & rheumarthritis & 3 \\
\hline 7 & 34 & M & 84 & Trunk & -19.92970319 & 61.91409025 & biliary tract disorder & 3 \\
\hline 8 & 68 & M & 60 & Extremities & 36.99828277 & -27.63658318 & bursitis & 3 \\
\hline 9 & 77 & $\mathrm{M}$ & 346 & Trunk & 21.42662403 & -54.81110386 & psoriasiform dermatitis & 3 \\
\hline 10 & 67 & $\mathrm{~F}$ & 23 & Mucosa & 20.55968805 & -15.27295948 & psoriasiform dermatitis & 3 \\
\hline 11 & 75 & $\mathrm{~F}$ & 42 & Mucosa & 140.8945355 & -1.171878513 & interstitial pneumonia & 2 \\
\hline 12 & 54 & $\mathrm{~F}$ & 126 & Mucosa & 3.937154859 & -15.87606189 & diarrhea & 2 \\
\hline 13 & 62 & $\mathrm{~F}$ & 121 & Trunk & -36.4146062 & -48.4018883 & adrenal insufficiency & 2 \\
\hline 14 & 81 & $\mathrm{~F}$ & 77 & Extremities & -22.47729919 & -70.94219363 & thyroid dysfunction & 2 \\
\hline 15 & 67 & $\mathrm{M}$ & 132 & Extremities & 34.16606428 & 6.260637076 & hypophisitis & 2 \\
\hline 16 & 61 & $\mathrm{~F}$ & 21 & Extremities & -8.525671721 & -3.390285544 & thyroid dysfunction & 2 \\
\hline 17 & 65 & $\mathrm{M}$ & 221 & Extremities & -76.45520955 & 2.912773985 & thyroid dysfunction & 2 \\
\hline 18 & 69 & $\mathrm{~F}$ & 62 & Mucosa & -33.29336941 & 25.19612409 & thyroid dysfunction & 2 \\
\hline 19 & 76 & $\mathrm{~F}$ & 210 & Extremities & 28.36775442 & 103.3236074 & hypophisitis & 2 \\
\hline 20 & 33 & $\mathrm{~F}$ & 84 & Extremities & 77.54691463 & 0.457742932 & hypophisitis & 1 \\
\hline 21 & 77 & $\mathrm{~F}$ & 143 & Mucosa & -41.53950593 & -24.59772023 & adrenal insufficiency & 1 \\
\hline 22 & 62 & $\mathrm{M}$ & 92 & Other/Unknown & -47.99843144 & -11.35075991 & adrenal insufficiency & 1 \\
\hline 23 & 60 & $\mathrm{M}$ & N.A. & Trunk & -4.542122094 & -16.16551984 & N.A. & N.A. \\
\hline 24 & 34 & $\mathrm{~F}$ & N.A. & Extremities & -29.55718265 & 0.254223734 & N.A. & N.A. \\
\hline 25 & 61 & $\mathrm{~F}$ & N.A. & Mucosa & -47.76858108 & -80.42942923 & N.A. & N.A. \\
\hline 26 & 76 & $\mathrm{M}$ & N.A. & Trunk & -26.66666667 & -19.75628258 & N.A. & N.A. \\
\hline 27 & 79 & $\mathrm{M}$ & N.A. & Head/Neck & -8.395159834 & -18.21571007 & N.A. & N.A. \\
\hline 28 & 84 & $\mathrm{~F}$ & N.A. & Trunk & 4.932359223 & -2.175956805 & N.A. & N.A. \\
\hline 29 & 68 & $\mathrm{M}$ & N.A. & Extremities & 14.99180027 & -24.6852751 & N.A. & N.A. \\
\hline 30 & 93 & $\mathrm{M}$ & N.A. & Extremities & 1.939261048 & 2.454790431 & N.A. & N.A. \\
\hline 31 & 74 & $\mathrm{M}$ & N.A. & Trunk & -22.07524444 & 1.421405214 & N.A. & N.A. \\
\hline 32 & 54 & $\mathrm{M}$ & N.A. & Mucosa & -4.745006346 & -10.67711099 & N.A. & N.A. \\
\hline 33 & 67 & $\mathrm{M}$ & N.A. & Extremities & 3.416168176 & -40.954426 & N.A. & N.A. \\
\hline 34 & 70 & $\mathrm{M}$ & N.A. & Head/Neck & -12.31934772 & -26.09262273 & N.A. & N.A. \\
\hline 35 & 65 & $\mathrm{M}$ & N.A. & Mucosa & 11.10220568 & 1.102732526 & N.A. & N.A. \\
\hline
\end{tabular}




\begin{tabular}{llllcllll}
36 & 79 & F & N.A. & Extremities & -30.35604275 & 14.93305921 & N.A. & N.A. \\
37 & 72 & M & N.A. & Mucosa & -4.40651476 & 194.1480243 & N.A. & N.A. \\
38 & 89 & M & N.A. & Other/Unknown & -0.721084093 & -26.81119932 & N.A. & N.A. \\
39 & 55 & F & N.A. & Mucosa & 3.390383922 & 44.79528142 & N.A. & N.A. \\
40 & 82 & M & N.A. & Mucosa & 46.17573498 & 33.3969329 & N.A. & N.A. \\
41 & 73 & M & N.A. & Extremities & -19.03589077 & -7.105634886 & N.A. & N.A. \\
42 & 62 & F & N.A. & Trunk & -58.26412844 & 31.31318466 & N.A. & N.A. \\
43 & 31 & F & N.A. & Head/Neck & -15.77316618 & 9.483456384 & N.A. & N.A. \\
44 & 64 & M & N.A. & Mucosa & 8.078227869 & 20.27538744 & N.A. & N.A. \\
45 & 77 & F & N.A. & Mucosa & -42.70869091 & -27.32383979 & N.A. & N.A. \\
46 & 65 & F & N.A. & Extremities & -16.12972729 & -24.15391828 & N.A. & N.A. \\
\hline
\end{tabular}

Serum levels of sCD163 and CXCL5 from each patient $(n=46)$ on days 0 and 42 were examined by ELISA.

in patients with irAEs (Supplementary Figure 1). Notably, immunofluorescence staining revealed that CD163+ macrophages were distributed in POSTN-expressing areas (Figure 3A), suggesting that POSTN in melanoma can stimulate CD163+ macrophages to produce CXCL5 (Figure 3B).
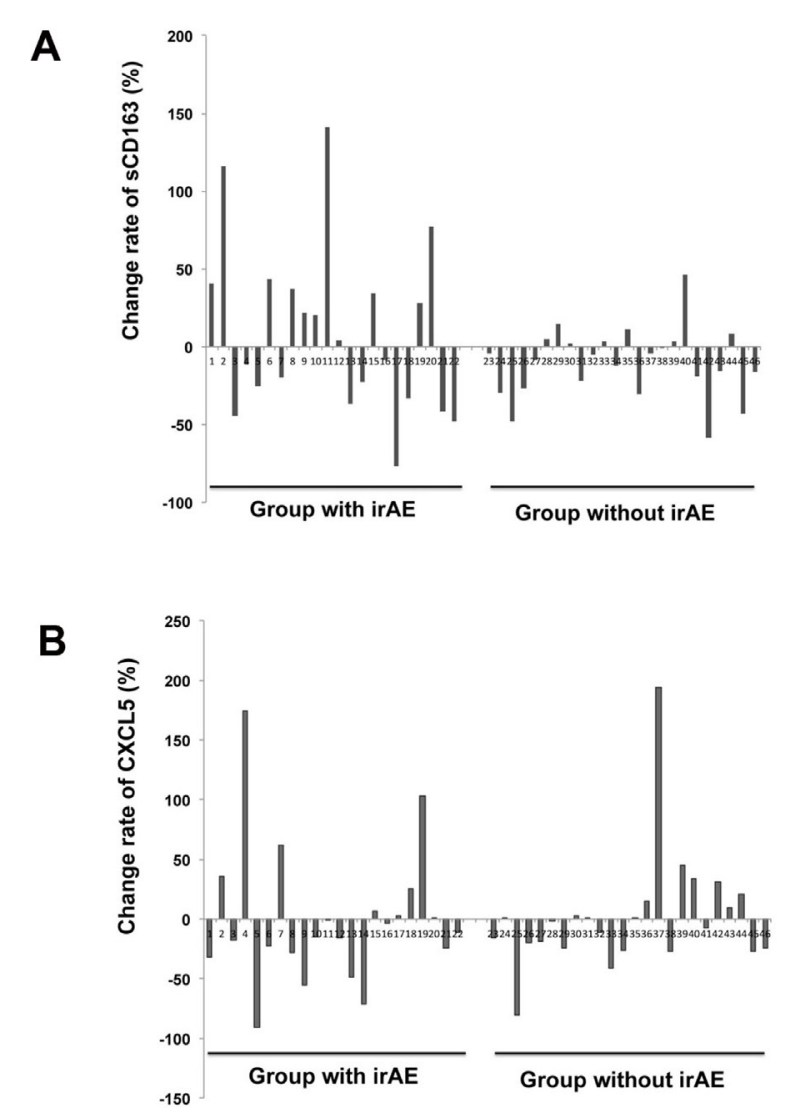

\section{DISCUSSION}

Because of nivolumab's higher efficacy than other anti-melanoma drugs (e.g., ipilimumab and dacarbazine) $[1,21]$, and because it induces a longer duration of anti-tumor response than BRAF/MEK inhibitors (e.g.,
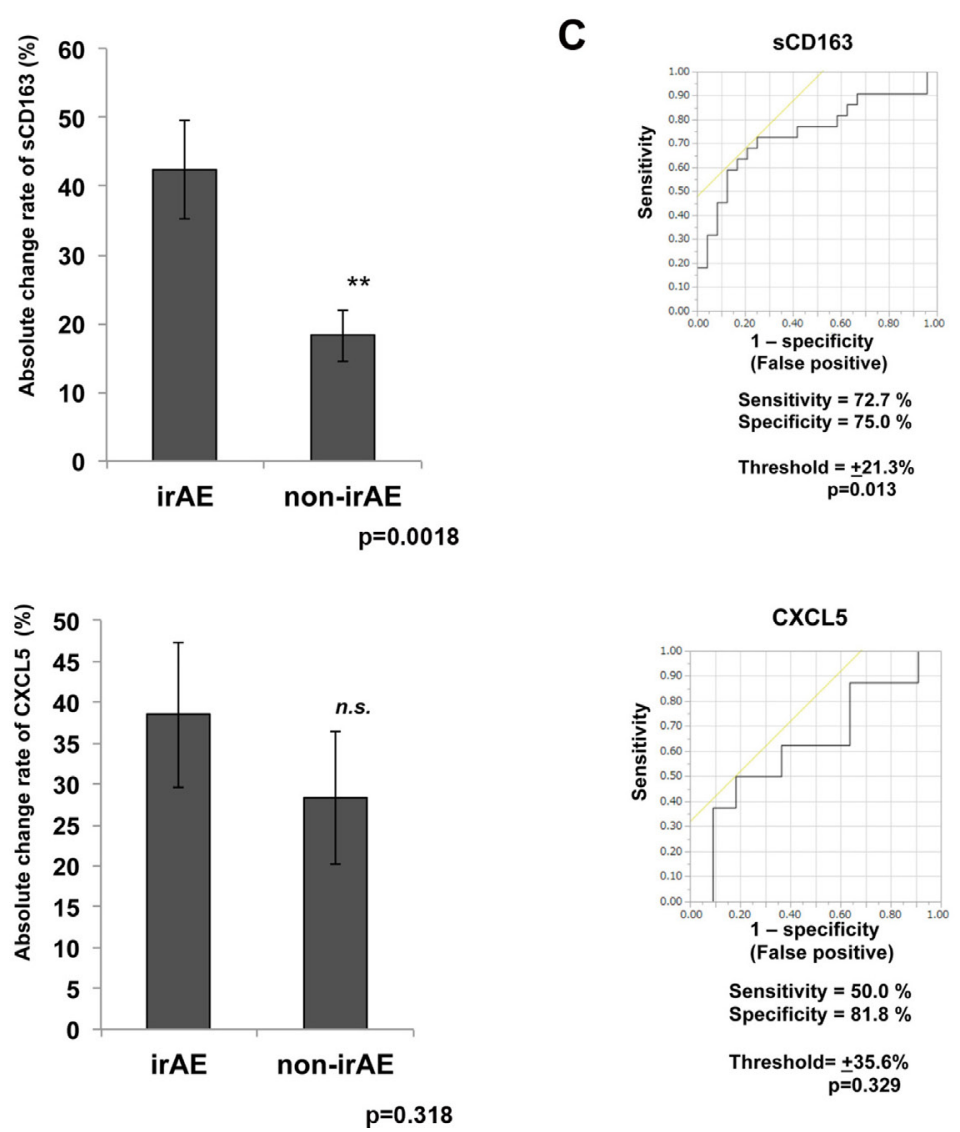

Figure 1: ROC curve of serum levels of SCD163 or CXCL5. The change in serum levels of SCD163 (A) and CXCL5 (B) from each patient ( $n=46)$ with or without irAE on day 42 . The ROC curve was applied to calculate the cut-off values of the sCD163 or CXCL5 serum levels and AUC $(\mathbf{C})$. The cut-off point was determined using Youden's index. 
Table 2: Serum levels of sCD163 and CXCL5 in each irAE

\begin{tabular}{|c|c|c|c|c|c|c|}
\hline Case & $\operatorname{ir} \mathbf{A E}$ & Grade & sCD163 (ng/ml) & sCD163 & CXCL5 (pg/ml) & CXCL5 \\
\hline 1 & hypophisitis & 4 & 115.782943 & + & 35.56162835 & + \\
\hline 15 & hypophisitis & 2 & 34.16606428 & + & 6.260637076 & + \\
\hline 19 & hypophisitis & 2 & 28.36775442 & + & 103.3236074 & + \\
\hline 20 & hypophisitis & 1 & 77.54691463 & + & 0.457742932 & + \\
\hline 14 & thyroid dysfunction & 2 & -22.47729919 & - & -70.94219363 & - \\
\hline 16 & thyroid dysfunction & 2 & -8.525671721 & - & -3.390285544 & - \\
\hline 17 & thyroid dysfunction & 2 & -76.45520955 & - & 2.912773985 & + \\
\hline 18 & thyroid dysfunction & 2 & -33.29336941 & - & 25.19612409 & + \\
\hline 13 & adrenal insufficiency & 2 & -36.4146062 & - & -48.4018883 & - \\
\hline 21 & adrenal insufficiency & 1 & -41.53950593 & - & -24.59772023 & - \\
\hline 22 & adrenal insufficiency & 1 & -47.99843144 & - & -11.35075991 & - \\
\hline 9 & psoriasiform dermatitis & 3 & 21.42662403 & + & -54.81110386 & - \\
\hline 10 & psoriasiform dermatitis & 3 & 20.55968805 & + & -15.27295948 & - \\
\hline 3 & interstitial pneumonia & 4 & -11.4747151 & - & 174.0938997 & + \\
\hline 11 & interstitial pneumonia & 2 & 140.8945355 & + & -1.171878513 & - \\
\hline 2 & hepatitis & 4 & -44.23121476 & - & -17.57753725 & - \\
\hline 4 & radiation dermatitis & 3 & 40.72768869 & - & -31.97872991 & - \\
\hline 5 & $\begin{array}{c}\text { chronic inflammatory } \\
\text { demyelinating polyneuropathy }\end{array}$ & 3 & -25.44524995 & - & -91.17233749 & - \\
\hline 6 & rheumarthritis & 3 & 43.18056154 & - & -22.79478559 & - \\
\hline 7 & biliary tract disorder & 3 & -19.92970319 & + & 61.91409025 & + \\
\hline 8 & bursitis & 3 & 36.99828277 & - & -27.63658318 & - \\
\hline
\end{tabular}

Serum levels of sCD163 and CXCL5 from each patient $(n=46)$ on days 0 and 42 were examined by ELISA in each irAE.

vemurafenib, dabrafenib, and trametinib) [22, 23], oncologists have been particularly interested in combining nivolumab with agents that enhance the anti-tumor immune response in patients with metastatic melanoma $[3,8,24$, 25]. The efficacy of nivolumab is significantly increased when combined with ipilimumab (57.7\%); however, unfortunately, the rate of severe treatment-related AEs (Grade 3 or 4 ) is also significantly increased with this particular combination $(55.0 \%)[2,24]$. These findings suggest the importance of predicting irAEs to avoid severe complications using simple methods. Indeed, Fujisawa et al. [26] reported that the decrease of lymphocytes fraction could predict irAEs caused by nivolumab, but only in the very short period before irAE development. Since the interval for nivolumab administration is 2 or 3 weeks for melanoma patients, other prognostic biomarkers are needed for long intervals.

CD163 is a member of the scavenger receptor cysteine-rich family, and is exclusively expressed on cells of monocyte/macrophage lineage [19, 27]. In lesions of various skin cancers, CD163+ macrophages are a main component of TILs that can produce various chemokines by stimulating cancer-specific stromal factors such as POSTN, IL-4, and RANKL $[11,14,28]$. sCD163 is an activation marker for $\mathrm{CD}_{163^{+}}$tissue macrophages that is present in the serum as a result of proteolytic shedding [19]. Notably, serum sCD163 levels increase in autoimmune diseases such as atherosclerosis, rheumatoid arthritis, pemphigus vulgaris, and bullous pemphigoid [17, 19, 28], and reflect disease activity [19]. In aggregate, $\mathrm{sCD} 163$ could be a macrophage activation marker to predict the production of cancer-specific chemokines from TAMs in different skin cancers.

CXCL5 is a chemokine that can recruit neutrophils and CXCR2+ myeloid cells, including myeloid-derived suppressor cells (MDSCs) and monocytes that can be a precursor of TAMs in tumor-bearing hosts [29]. Recently, Katoh et al. [29] reported that the inhibition of CXCR2+ MDSC recruitment into colonic mucosa and tumors dramatically suppress colonic inflammation, suggesting that CXCR2+ MDSCs are essential for colitis-associated cancer. In another report, Steele et al. [30] suggested that CXCR2 signaling in the myeloid component can promote pancreatic tumorigenesis and is required for pancreatic cancer metastasis. Zhang et al. [31] reported that the depletion of MDSCs decreased IL-17A and IL- 
$1 \beta$ production, leading to therapeutic effects of arthritis in a mouse model, suggesting the importance of proinflammatory MDSCs in autoimmune disease. MDSCs are a heterogeneous population of cells that can be induced by tumor-associated inflammation, including autoimmune related cytokines such as IL-1 $\beta$ and IL-6 [32]. Because POSTN is an extracellular matrix protein that can be detected in the dermis of various skin inflammatory disorders including autoimmune disease such as scleroderma, pemphigus vulgaris, and bullous pemphigoid $[17,33]$, POSTN in melanoma might stimulate MDSCs to further produce chemokines such as CXCL5.

In this report, we analyzed the serum levels of sCD163 and CXCL5 in 46 cases of advanced melanoma treated with nivolumab. The tumor stroma of melanoma had increased expression of POSTN upon administration with nivolumab, and POSTN stimulated TAMs to release CXCL5 [14]. The increased absolute value of serum levels of SCD163 and CXCL5 in a patient who develops adverse nivolumab-induced, immune-related events is presumably related to POSTN-stimulated TAM activation. Therefore, in the setting of nivolumab therapy, sCD163 and CXCL5 serum levels may serve as valuable predictors of irAEs [8, 20]; thus, we measured serum levels of sCD163 and CXCL5 at day 0 (immediately before nivolumab administration) and day 42. In addition, as presented in Table 2, the change in levels of sCD163 and CXCL5 tend to differ in each irAE.
Although we could not determine the precise reasons, one possible explanation of this observation is that the time point of TAM activation differs in each irAE. Because the recruitment of effector cells to target organs is indispensable for the development of irAEs, and since one of the main sources of chemokines are macrophages, the activation of macrophages is important for the development of irAEs. Decreased serum levels of sCD163, especially in cases of thyroid dysfunction or adrenal insufficiency, indicates that CD163+ macrophages have been activated and effector cells have been recruited to the target organ, both of which underly the development of irAEs by nivolumab. Therefore, serum levels of sCD163 were significantly increased or decreased in patients who developed AEs $(p=0.013)$. This phenomenon could be explained by the heterogeneity of TAMs, which consist of macrophages in various phases of activation [34]. Indeed, the serum levels of CXCL5 correlate with autoimmune diseases such as multiple sclerosis, rheumatoid arthritis, glomerulonephritis, pemphigus vulgaris, and bullous pemphigoid with several inflammation factors [16-18, 35], though the main sources of CXCL5 is not fully investigated in each reports. Thus, although there was no significant difference in CXCL5 levels, the change rate of CXCL5 may be a useful marker for predicting the organs affected by irAEs. Because this was a pilot study, future independent studies with a larger patient cohort are needed to confirm our findings.

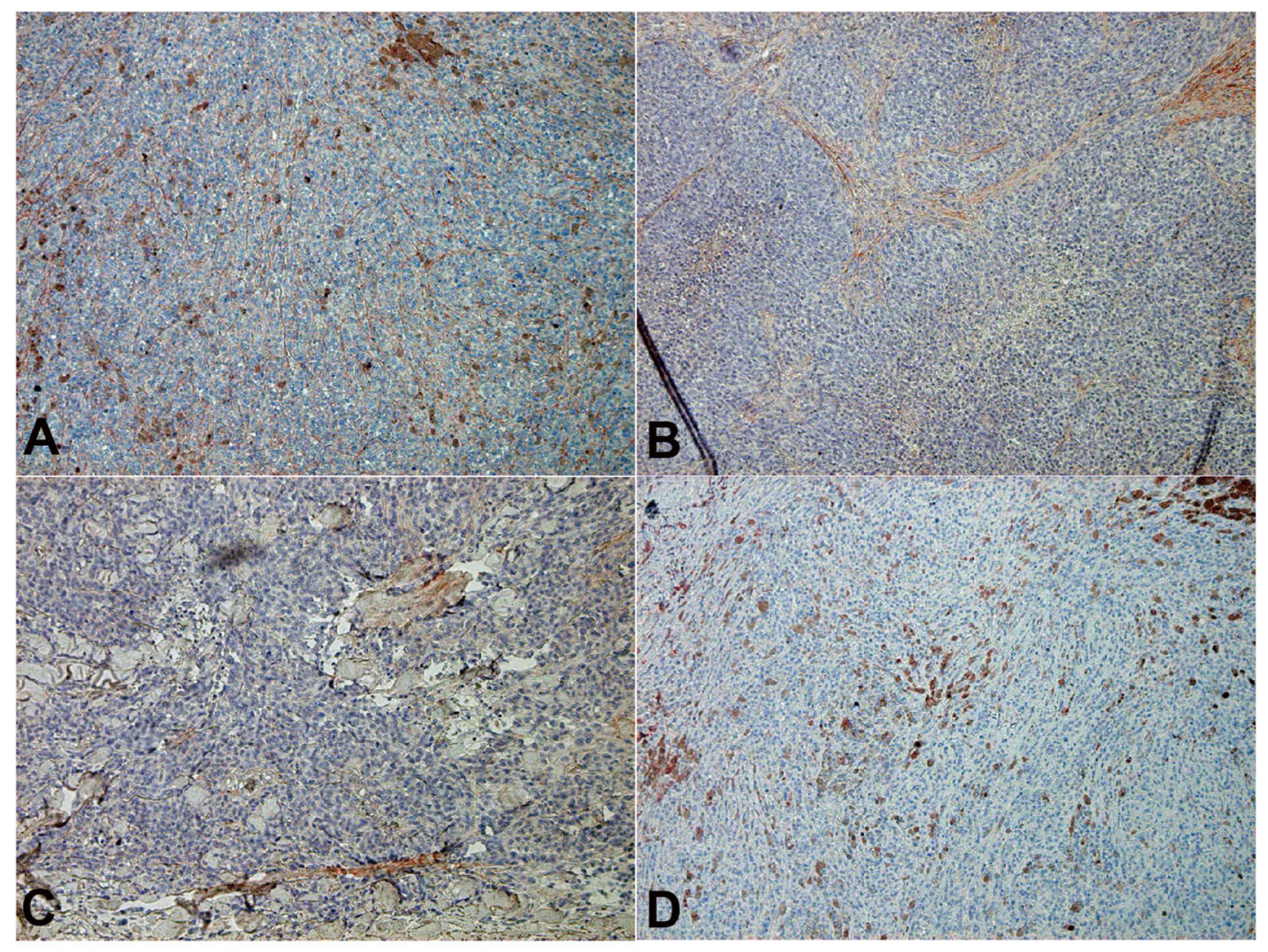

Figure 2: POSTN expression in in-transit melanoma. Sections of in-transit melanoma from patients with irAE after nivolumab (A) and before nivolumab administration (B), and patients without irAE (C) were deparaffinized and stained using anti-POSTN (A, B, C) or anti-CD163 Abs (D). The signal was developed with 3-amino-9-ethylcarbazole. 
Table 3: Semi-quantitative analysis of immunohistochemical staining of POSTN

\begin{tabular}{|c|c|c|c|}
\hline & ir AE grade & Pre treatment & Post treatment \\
\hline Case 1 & 4 & + & +++ \\
\hline Case 4 & 3 & + & +++ \\
\hline Case 23 & $\mathbf{0}$ & ++ & + \\
\hline Case 28 & 0 & ++ & + \\
\hline \multirow[t]{5}{*}{ Case 41} & $\mathbf{0}$ & + & + \\
\hline & & - & negative \\
\hline & & + & weak \\
\hline & & ++ & moderate \\
\hline & & +++ & intense \\
\hline
\end{tabular}

The intensity of immunohistochemical staining of POSTN was scored on a semi-quantitative scale.

\section{Ethics statement for animal and human experiments}

The protocol for the human study was approved by the ethics committee of Tohoku University Graduate School of Medicine, Sendai, Japan (Permit No: 2017-1-377).

\section{PATIENTS AND METHODS}

Data from patients who were treated with nivolumab were collected from six clinical sites in Japan. Patients were eligible if they had unresectable stage III melanoma, if their tumor was resectable but they had

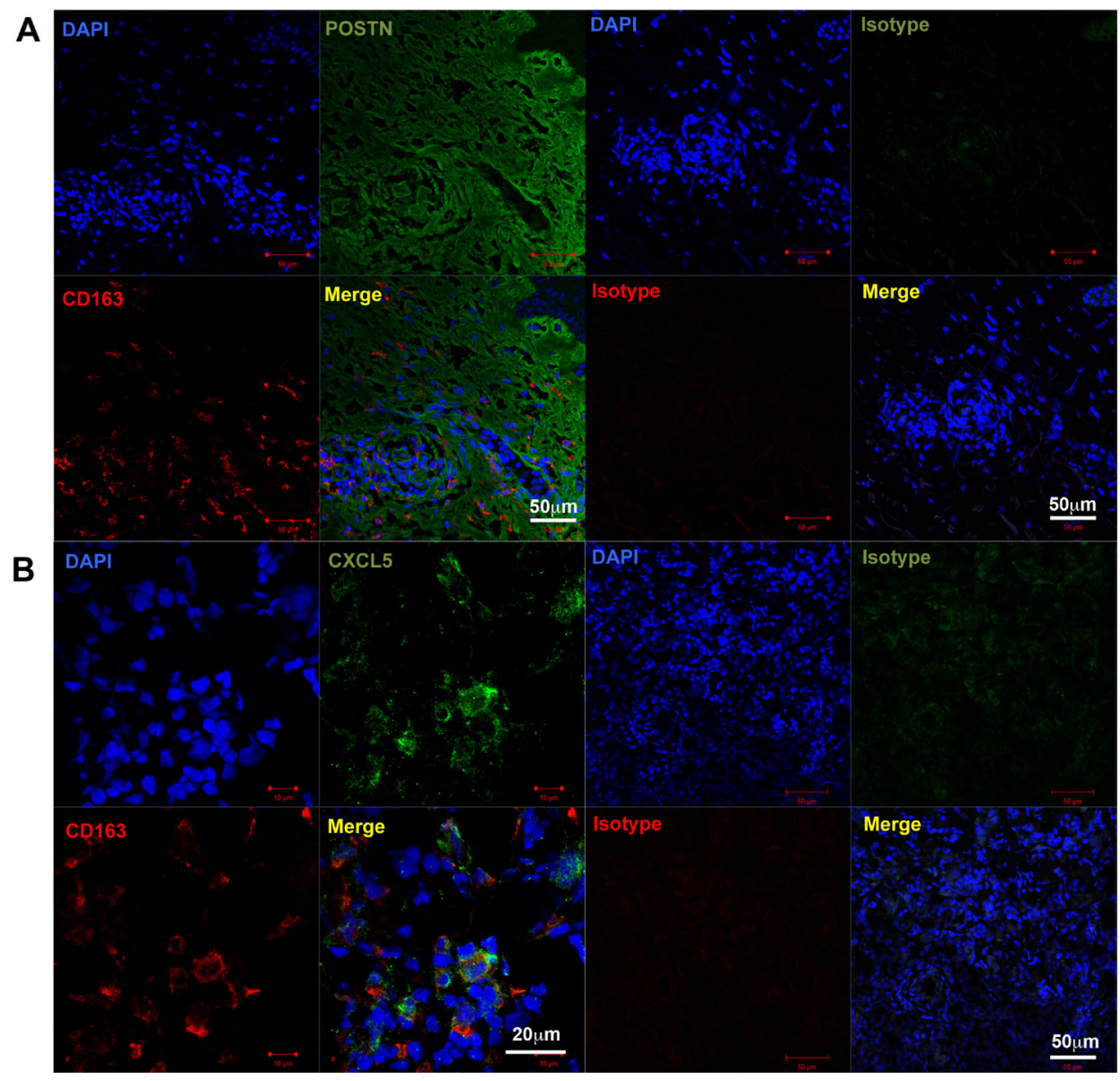

Figure 3: CD163+ cells in in-transit melanoma. Immunofluorescence staining of in-transit melanoma for (A) POSTN (green), CD163 (red), and DAPI (blue, nucleus), and (B) CXCL5 (green), CD163 (red), and DAPI (blue, nucleus). A merged image is also shown. Merged green and red stain yellow. Representative specimens from five cases are shown. 
declined resection, or if they had stage IV melanoma with accessible cutaneous, subcutaneous, and/or nodal lesions (patients were staged according to the AJCC Staging Manual, 7th Edition, 2011). All patients received $2 \mathrm{mg} /$ $\mathrm{kg}$ nivolumab followed by a 3 -week rest period or $3 \mathrm{mg} /$ $\mathrm{kg}$ nivolumab followed by 2 weeks of rest, both of which are the approved dosing schedule in Japan. Serum from patients was obtained on days 0 and 42 . In our 46 cases, case 5 was administered systemic steroid on day 28 , and the serum levels in this case may have been affected by systemic steroid. The other two cases (cases 10 and 16) were treated with topical steroid (case 10) or levothyroxine sodium hydrate (case 16).

\section{Serum levels of sCD163 and CXCL5}

On days 0 and 42 after nivolumab administration, we analyzed the serum levels of soluble sCD163 and CXCL5 by enzyme-linked immunoassay (ELISA) according to the manufacturer's protocol (R\&D Systems, Minneapolis, MN, USA). The serum levels on day 42 were compared to baseline (day 0) and statistically analyzed.

\section{Reagents}

The following Abs were used for immunohistochemistry: mouse anti-human CD163 monoclonal (Novocastra, Tokyo, Japan) and rabbit polyclonal antiPOSTN (Abcam, Tokyo, Japan). The following Abs were used for immunofluorescence: mouse anti-human CD163 phycoerythrin-conjugated monoclonal antibody (R\&D Systems), rabbit polyclonal POSTN (Abcam), and Alexa Fluor 488-conjugated anti-rabbit goat IgG (Abcam).

\section{Tissue samples, immunohistochemistry, and immunofluorescence}

Archival formalin-fixed paraffin-embedded skin specimens were collected from five patients with in-transit advanced melanoma without irAEs, and from two patients with in-transit advanced melanoma with irAEs (Grades 2 and 3 hypophysitis), who were treated in the Department of Dermatology at Tohoku University Graduate School of Medicine. All patients gave written informed consent. The in-transit melanoma samples were processed for single staining of CD163a and POSTN, and the signal was developed with 3-amino-9-ethylcarbazole (Nichirei Bioscience, Tokyo, Japan). For cryosections, five cases of in-transit melanoma samples were frozen in optimal cutting temperature embedding medium (Sakura Finetek Japan Co. Ltd., Tokyo, Japan), and $6 \mu \mathrm{m}$ thick sections were fixed in cold acetone for $30 \mathrm{~min}$ and blocked in immunofluorescence buffer (phosphate-buffered saline, $5 \%$ bovine serum albumin). Thereafter, each section was incubated with the relevant antibodies. The slides were mounted in DAPI Fluoromount-G (Southern Biotech,
Birmingham, AL, USA) and examined using a Zeiss LSM 700 microscope equipped with a Spot digital camera.

\section{Statistical methods}

The receiver operating characteristic (ROC) curve was applied to calculate the cut-off values for the serum levels of sCD163 or CXCL5 and the area under the curve (AUC). The cut-off point was determined using Youden's index. The ROC curves were established to evaluate the serum levels of sCD163 and CXCL5 in patients who developed irAEs. For a single comparison between two groups, the Mann-Whitney $U$-test or Student's $t$-test was used. Correlation coefficients were determined using the Spearman's rank correlation test. The level of significance was set at $p<0.05$. All statistical analyses were performed using JMP 13.1 software (SAS Institute Inc., Tokyo, Japan).

\section{Author contributions}

Fujimura T designed the research study. Fujimura T, Sato Y, Tanita K, Yumi performed and analyzed the ELISA data. Sato Y performed the immunofluorescence. Sato Y and Tanita K performed the immunohistochemistry. Fujimura T, Kambayashi Y, Otsuka A, Fujisawa Y, Yoshino K, Matsushita S, Funakoshi T, Hata H, Yamamoto Y, Uchi H, Nonomura Y, Tanaka R, Aoki M, Imafuku K, Okuhira H, Furudate S and Hidaka T treated the patients and acquired the clinical data and samples. Fujimura $\mathrm{T}$ wrote the manuscript. Fujimura T and Aiba S supervised the study.

\section{ACKNOWLEDGMENTS}

We thank K. Miyata, Y. Otsuka, K Togashi, and M. Hosaka for performing the statistical analyses.

\section{CONFLICTS OF INTEREST}

This study was partially funded by Japan Agency for Medical Research and Development (2017-U-059), Ono Pharmaceutical Co., Ltd. and Bristol-Myers Squibb.

\section{REFERENCES}

1. Wolchok JD, Kluger H, Callahan MK, Postow MA, Rizvi NA, Lesokhin AM, Segal NH, Ariyan CE, Gordon RA, Reed K, Burke MM, Caldwell A, Kronenberg SA, et al. Nivolumab plus ipilimumab in advanced melanoma. N Engl J Med. 2013; 369:122-33.

2. Larkin J, Chiarion-Sileni V, Gonzalez R, Grob JJ, Cowey CL, Lao CD, Schadendorf D, Dummer R, Smylie M, Rutkowski P, Ferrucci PF, Hill A, Wagstaff J, et al. Combined Nivolumab and Ipilimumab or Monotherapy in Untreated Melanoma. N Engl J Med. 2015; 373:23-34. 
3. Topalian SL, Sznol M, McDermott DF, Kluger HM, Carvajal RD, Sharfman WH, Brahmer JR, Lawrence DP, Atkins MB, Powderly JD, Leming PD, Lipson EJ, Puzanov I, et al. Survival, durable tumor remission, and long-term safety in patients with advanced melanoma receiving nivolumab. J Clin Oncol. 2014; 32:1020-30.

4. Liniker E, Menzies AM, Kong BY, Cooper A, Ramanujam S, Lo S, Kefford RF, Fogarty GB, Guminski A, Wang TW, Carlino MS, Hong A, Long GV. Activity and safety of radiotherapy with anti-PD-1 drug therapy in patients with metastatic melanoma. OncoImmunology. 2016; 5:e1214788.

5. Fujimura T, Kambayashi Y, Furudate S, Hidaka T, Sato Y, Tanita K, Tono H, Tsukada A, Hashimoto A, Aiba S. Successful treatment of multiple in-transit melanomas on the leg with intensity-modulated radiotherapy and immune checkpoint inhibitors: report of two cases. J Dermatol. 2017; 44:592-95.

6. Fujimura T, Furudate S, Kakizaki A, Kambayashi Y, Haga T, Hashimoto A, Aiba S. Contact immunotherapy enhances the therapeutic effects of nivolumab in treating in-transit melanoma: two cases reports. J Dermatol. 2016; 43:686-89.

7. Gulati N, Carvajal RD, Postow MA, Wolchok JD, Krueger JG. Definite regression of cutaneous melanoma metastases upon addition of topical contact sensitizer diphencyprone to immune checkpoint inhibitor treatment. Exp Dermatol. 2016; 25:553-54.

8. Fujimura T, Hidaka T, Kambayashi Y, Furudate S, Kakizaki A, Tono H, Tsukada A, Haga T, Hashimoto A, Morimoto R, Yamaguchi T, Takano T, Aiba S. Phase I study of nivolumab combined with IFN- $\beta$ for patients with advanced melanoma. Oncotarget. 2017; 8:71181-87. https://doi.org/10.18632/ oncotarget.17090.

9. Yue EW, Sparks R, Polam P, Modi D, Douty B, Wayland B, Glass B, Takvorian A, Glenn J, Zhu W, Bower M, Liu X, Leffet L, et al. INCB24360 (Epacadostat), a Highly Potent and Selective Indoleamine-2,3-dioxygenase 1 (IDO1) Inhibitor for Immuno-oncology. ACS Med Chem Lett. 2017; 8:486-91.

10. Noy R, Pollard JW. Tumor-associated macrophages: from mechanisms to therapy. Immunity. 2014; 41:49-61.

11. Fujimura T, Kambayashi Y, Furudate S, Asano M, Kakizaki A, Aiba S. Receptor Activator of NF- $\kappa B$ Ligand Promotes the Production of CCL17 from RANK+ M2 Macrophages. J Invest Dermatol. 2015; 135:2884-87.

12. Komohara Y, Fujiwara Y, Ohnishi K, Takeya M. Tumorassociated macrophages: Potential therapeutic targets for anti-cancer therapy. Adv Drug Deliv Rev. 2016; 99:80-185.

13. Caronni N, Savino B, Bonecchi R. Myeloid cells in cancerrelated inflammation. Immunobiology. 2015; 220:249-53.

14. Furudate S, Fujimura T, Kakizaki A, Kambayashi Y, Asano M, Watabe A, Aiba S. The possible interaction between periostin expressed by cancer stroma and tumor-associated macrophages in developing mycosis fungoides. Exp Dermatol. 2016; 25:107-12.
15. Fukuda K, Sugihara E, Ohta S, Izuhara K, Funakoshi T, Amagai M, Saya H. Periostin Is a Key Niche Component for Wound Metastasis of Melanoma. PLoS One. 2015; 10:e0129704.

16. Rumble JM, Huber AK, Krishnamoorthy G, Srinivasan A, Giles DA, Zhang X, Wang L, Segal BM. Neutrophil-related factors as biomarkers in EAE and MS. J Exp Med. 2015; 212:23-35.

17. Fujimura T, Kakizaki A, Furudate S, Aiba S. A possible interaction between periostin and CD163+skin-resident macrophages in pemphigus vulgaris and bullous pemphigoid. Exp Dermatol. 2017; 26:1193-98.

18. Buckland J. Rheumatoid arthritis: citrullination alters the inflammatory properties of chemokines in inflammatory arthritis. Nat Rev Rheumatol. 2014; 10:446.

19. Van Gorp H, Delputte PL, Nauwynck HJ. Scavenger receptor CD163, a Jack-of-all-trades and potential target for celldirected therapy. Mol Immunol. 2010; 47:1650-60.

20. Fujimura T, Kambayashi Y, Furudate S, Kakizaki A, Hidaka T, Haga T, Hashimoto A, Morimoto R, Aiba S. Isolated adrenocorticotropic hormone deficiency possibly caused by nivolumab in a metastatic melanoma patient. J Dermatol. 2017; 44:e13-14.

21. Robert C, Long GV, Brady B, Dutriaux C, Maio M, Mortier L, Hassel JC, Rutkowski P, McNeil C, Kalinka-Warzocha E, Savage KJ, Hernberg MM, Lebbé C, et al. Nivolumab in previously untreated melanoma without BRAF mutation. $\mathrm{N}$ Engl J Med. 2015; 372:320-30.

22. McArthur GA, Chapman PB, Robert C, Larkin J, Haanen JB, Dummer R, Ribas A, Hogg D, Hamid O, Ascierto PA, Garbe C, Testori A, Maio M, et al. Safety and efficacy of vemurafenib in BRAF(V600E) and BRAF(V600K) mutation-positive melanoma (BRIM-3): extended follow-up of a phase 3, randomised, open-label study. Lancet Oncol. 2014; 15:323-32.

23. Long GV, Stroyakovskiy D, Gogas H, Levchenko E, de Braud F, Larkin J, Garbe C, Jouary T, Hauschild A, Grob JJ, Chiarion Sileni V, Lebbe C, Mandalà M, et al. Combined BRAF and MEK inhibition versus BRAF inhibition alone in melanoma. N Engl J Med. 2014; 371:1877-88.

24. Weber JS, Gibney G, Sullivan RJ, Sosman JA, Slingluff CL Jr, Lawrence DP, Logan TF, Schuchter LM, Nair S, Fecher L, Buchbinder EI, Berghorn E, Ruisi M, et al. Sequential administration of nivolumab and ipilimumab with a planned switch in patients with advanced melanoma (CheckMate 064): an open-label, randomised, phase 2 trial. Lancet Oncol. 2016; 17:943-55.

25. Kakizaki A, Fujimura T, Furudate S, Kambayashi Y, Yamauchi T, Yagita H, Aiba S. Immunomodulatory effect of peritumorally administered interferon-beta on melanoma through tumor-associated macrophages. OncoImmunology. 2015; 4:e1047584.

26. Fujisawa Y, Yoshino K, Otsuka A, Funakoshi T, Fujimura T, Yamamoto Y, Hata H, Gosho M, Tanaka R, Yamaguchi 
K, Nonomura Y, Hirai I, Furudate S, et al. Fluctuations in routine blood count might signal severe immunerelated adverse events in melanoma patients treated with nivolumab. J Dermatol Sci. 2017; 88:225-31.

27. Greisen SR, Møller HJ, Stengaard-Pedersen K, Hetland ML, Hørslev-Petersen K, Junker P, Østergaard M, Hvid M, Deleuran B. Macrophage activity assessed by soluble CD163 in early rheumatoid arthritis: association with disease activity but different response patterns to synthetic and biologic DMARDs. Clin Exp Rheumatol. 2015; 33:498-502.

28. Fujimura T, Kakizaki A, Furudate S, Kambayashi Y, Aiba S. Tumor-associated macrophages in skin: how to treat their heterogeneity and plasticity. J Dermatol Sci. 2016; 83:167-73.

29. Katoh H, Wang D, Daikoku T, Sun H, Dey SK, Dubois RN. CXCR2-expressing myeloid-derived suppressor cells are essential to promote colitis-associated tumorigenesis. Cancer Cell. 2013; 24:631-44.

30. Steele CW, Karim SA, Leach JD, Bailey P, UpstillGoddard R, Rishi L, Foth M, Bryson S, McDaid K, Wilson Z, Eberlein C, Candido JB, Clarke M, et al. CXCR2 Inhibition Profoundly Suppresses Metastases and Augments
Immunotherapy in Pancreatic Ductal Adenocarcinoma. Cancer Cell. 2016; 29:832-45.

31. Zhang H, Wang S, Huang Y, Wang H, Zhao J, Gaskin F, Yang N, Fu SM. Myeloid-derived suppressor cells are proinflammatory and regulate collagen-induced arthritis through manipulating Th17 cell differentiation. Clin Immunol. 2015; 157:175-86.

32. Fujimura T, Mahnke K, Enk AH. Myeloid derived suppressor cells and their role in tolerance induction in cancer. J Dermatol Sci. 2010; 59:1-6.

33. Yamaguchi Y, Ono J, Masuoka M, Ohta S, Izuhara K, Ikezawa Z, Aihara M, Takahashi K. Serum periostin levels are correlated with progressive skin sclerosis in patients with systemic sclerosis. Br J Dermatol. 2013; 168:717-25.

34. Gordon S, Martinez FO. Alternative activation of macrophages: mechanism and functions. Immunity. 2010; 32:593-604.

35. Disteldorf EM, Krebs CF, Paust HJ, Turner JE, Nouailles G, Tittel A, Meyer-Schwesinger C, Stege G, Brix S, Velden J, Wiech T, Helmchen U, Steinmetz OM, et al. CXCL5 drives neutrophil recruitment in TH17-mediated GN. J Am Soc Nephrol. 2015; 26:55-66. 